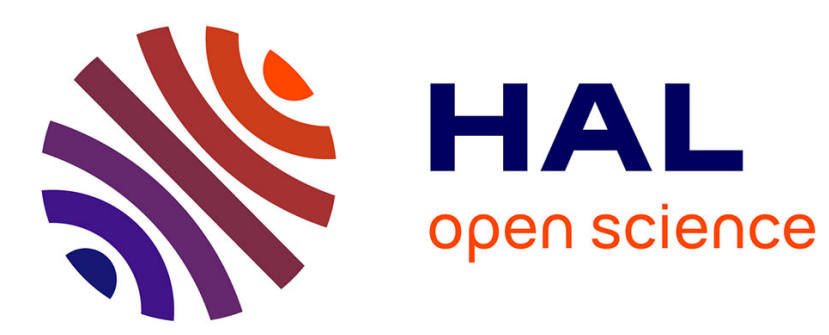

\title{
Rencontres avec l'enfermement. Une réflexion sur le soin en psychiatrie
}

\author{
Livia Velpry
}

\section{To cite this version:}

Livia Velpry. Rencontres avec l'enfermement. Une réflexion sur le soin en psychiatrie. Empan, 2019, 10.3917/empa.114.0044 . hal-02283114

\section{HAL Id: hal-02283114 \\ https://hal.science/hal-02283114}

Submitted on 10 Sep 2019

HAL is a multi-disciplinary open access archive for the deposit and dissemination of scientific research documents, whether they are published or not. The documents may come from teaching and research institutions in France or abroad, or from public or private research centers.
L'archive ouverte pluridisciplinaire HAL, est destinée au dépôt et à la diffusion de documents scientifiques de niveau recherche, publiés ou non, émanant des établissements d'enseignement et de recherche français ou étrangers, des laboratoires publics ou privés. 


\section{Rencontres avec l'enfermement.}

Une réflexion sur le soin en psychiatrie

A paraître dans EMPAN (2019)

\section{Livia Velpry}

Maîtresse de conférences en sociologie

Université Paris8 - CERMES3

https://liviavelpry.wordpress.com/

\section{Pré-print avant épreuves de l'article paru dans la revue EMPAN, $\mathbf{n}^{\circ} 114$, 2019/2}

Mots-clefs : psychiatrie; soin; unités pour malades difficiles; enfermement; violence ; santé mentale

Résumé: Mes recherches en sociologie m’ont conduite à étudier les situations de violence, les pratiques de contrainte et les unités sécurisées à l'hôpital psychiatrique, en parallèle de la montée de la démocratie sanitaire qui prône le respect des droits des patients et notamment de leur consentement. Ce texte parcourt ces terrains d'enquête au prisme de l'enfermement, dont les paradoxes illustrent les mutations en cours dans le monde de la psychiatrie.

La réflexion proposée dans ce texte a émergé au fil d'enquêtes sociologiques que j'ai menées dans le monde de la psychiatrie au cours des dix dernières années. Une recherche sur la gestion des situations de violence dans les unités d'hospitalisation en psychiatrie, entamée en 2006, m'a conduite à étudier les pratiques de contrainte et d'enfermement en santé mentale. Alors que les régulations juridiques et administratives réaffirmaient que la relation de soin devait être contractuelle et consentie, ces pratiques devenaient de plus en plus difficiles à justifier. On discutait des règles qui devaient encadrer leur usage. Or, à cette même période, les politiques publiques tendaient à développer les unités sécurisées et la spécialisation des soins qui y étaient dispensés. Dans les pages qui suivent, je reviens sur ces paradoxes de l'enfermement pour illustrer des mutations du monde de la psychiatrie.

De la violence à l'enfermement

Dans les services de psychiatrie publique où je me rends, au milieu des années 2000, pour y réaliser observations et entretiens, la violence préoccupe. Entre deux faits divers, les témoignages des soignants affluent. Beaucoup éprouvent le sentiment que la violence fait partie de leur quotidien. Ils y voient plusieurs explications. Certains rappellent que la maladie mentale peut produire des états qui sont intrinsèquement violents, mais aussi que l'environnement institutionnel génère, lui aussi, sa propre violence. Ces effets se cumulent. Autrement dit, la violence fait partie de l'ordinaire quand on s'occupe de troubles mentaux. Pour ces professionnels, si l'on en parle de plus en plus, c'est parce 
que la société d'aujourd'hui tolère moins cette violence, dans son effort de lisser les rapports sociaux. Les soignants des plus jeunes générations, qui n’ont pas été formés aux spécificités de la psychiatrie, sont régulièrement cités en exemple. D’autres - ou parfois les mêmes - mettent en cause la disparition de l'asile. Son caractère contenant assurait une fonction de sécurisation; en outre, il avait un effet apaisant sur certaines pathologies. L'enfermement, en d'autres termes, avait ses vertus. Certes les rapports entre soignants et patients y étaient plus musclés, mais on y était protégé. C'est au nom de cette protection que des services sont dits "ouverts» alors même que la porte d'entrée y est fermée. Elle est ouverte, mais sur demande.

\section{L'enfermement, survivance de l'asile}

Pourtant, la psychiatrie publique définit son projet résolument contre l'enfermement, au moins depuis les années 1960. L'enfermement, c'est l'asile, c'est le passé. La psychiatrie aujourd'hui s'ancre dans la communauté et évite de rompre la continuité de la vie de la personne comme de la prise en charge. Peu importe que jusqu'aux années 1990 ce discours soit en partie contredit, dans les faits, par un nombre de lits d'hospitalisation qui reste conséquent, des séjours encore longs, et un rôle central de l'hôpital dans le mode de financement du système. La tendance est à l'ouverture. Parler d'enfermement, c'est aussi convoquer une psychiatrie dont l'objet est la maladie mentale. Or, depuis les années 1990, le terme de santé mentale est devenu l'euphémisme privilégié. En parlant de problèmes de santé mentale, on élargit le spectre des troubles. La maladie mentale est reléguée à une extrémité, celle de la folie. La chronicité s'estompe ; certains troubles se soignent. Les soins en santé mentale mettent en avant l'ambulatoire et la réduction de la stigmatisation.

Que devient alors l'enfermement en psychiatrie ? Est-il voué à disparaître ? Va-t-il persister non plus comme un principe général, mais uniquement dans des cas précis, et alors lesquels ? S'il doit être réservé à certains, à quel titre?

\section{Mues de l'enfermement en psychiatrie}

Je poursuis mes efforts pour comprendre ces discours où la violence associée aux troubles mentaux et à la psychiatrie semble si largement ressentie et si difficile à assumer. A la limite du spectre de la santé mentale, à l'endroit où les troubles résistent à l'euphémisation - parce que les crises qui surviennent ne peuvent être ignorées, parce que la violence qui les accompagne perturbe trop fortement l'environnement, parce que l'atteinte aux personnes est visible -, l'enfermement conserve une légitimité. Dans les années 1990, la chambre d'isolement est peu à peu reconnue comme outil de réponse à la crise, requérant une prescription médicale ${ }^{1}$. Avoir une chambre d'isolement par unité, parfois deux, devient la norme dans les années 2000. Au même moment, dans les établissements hospitaliers, on discute de l'opportunité de disposer d'une unité fermée mutualisée pour les patients qui présenteraient des comportements violents. Les unités commencent à distinguer des parties ouvertes et des parties fermées. Ces dernières sont

\footnotetext{
${ }^{1}$ L'encadrement juridique et administratif de l'usage de la chambre d'isolement prend la forme de préconisations dans la circulaire dite «Veil » du 19 juillet 1993 - qui en stabilise la définition. Il se poursuit par plusieurs recommandations de bonne pratique, avant son inscription dans l'article 72 de la loi du 26 janvier 2016.
} 
destinées aux patients hospitalisés sans leur consentement ou les patients détenus. Elles sont la condition pour que les patients hospitalisés librement profitent d'une unité ouverte, comme ils en ont le droit. Est-ce que cela va vraiment de soi ? Quand j'allais dans les unités d'hospitalisation des années 1990, certaines étaient ouvertes et d'autres fermées, selon la politique du chef de service. Par contre, le statut d'hospitalisation du patient n'entraînait pas de différence de traitement. Est-ce à dire, alors, que les motifs de l'enfermement changent? Que l'enfermement se développe?

En tout cas, même si sa justification est floue, entre sécurisation, respect de la législation et visée thérapeutique, on réserve l'enfermement aux situations de dernier recours, à la dernière extrémité. Je décide d'explorer cet usage en me rendant dans des lieux où l'enfermement est assumé. Ces lieux de la marge accueillent quelques centaines de patients quand la violence est telle qu'ils « ne peuvent plus être accueillis dans les unités ordinaires », selon la mission qui leur est assignée depuis leur création en 1911. Leur désignation sous le terme d'« unités pour malades difficiles » (UMD) porte elle aussi bien haut l'euphémisme.

\section{Psychiatrie à l'ancienne}

Les UMD constituent en quelque sorte un reliquat de l'asile. Les dernières ont été créées dans les années 50 et elles semblent avoir été oubliées du processus de réforme psychiatrique. Elles étaient cinq en 2010 et représentaient une part négligeable de la capacité d'hospitalisation en psychiatrie. En cela, la France se distingue de ses voisins. Au Royaume-Uni, par exemple, l'hospitalisation psychiatrique a été réorganisée dans les années 1990 selon un gradient de fermeture : il existe des unités de moyenne sécurité et des unités de haute sécurité. L'UMD est une institution atypique en Europe parce qu'elle accueille à la fois des patients qui perturbent trop les services de psychiatrie ordinaires, des personnes déclarées irresponsables pour le crime qu'elles ont commis et des détenus ayant besoin d'être hospitalisés. Pour cette raison, elle constitue une forme d'articulation originale entre enfermement, dangerosité et soin.

Quand les soignants parlent de l'UMD, que ce soit depuis l'extérieur ou l'intérieur, leur description est très polarisée, entre survivance de l'asile et expertise de la dangerosité. Une psychiatre me raconte ses déboires lorsqu'elle décide de baisser les dosages de ses prescriptions, dans l'unité pour malades difficiles où elle travaille. L'équipe infirmière résiste et va ouvertement jusqu'au rapport de force. Elle s'est déjà trouvée coincée dans la cuisine, menacée par certains infirmiers de «faire monter» les patients si elle persistait à baisser le dosage de ses prescriptions. Elle décrit une unité au fonctionnement disciplinaire sévère assumé, loin des unités d'hospitalisation aujourd'hui. Dans ces dernières, cependant, certaines de ces pratiques persistent sans doute, édulcorées.

\section{«Ici ils sortent, on a l'impression d'être efficace »}

Quel rôle joue exactement l'enfermement dans les unités pour malades difficiles ? Pour les soignants qui y travaillent, il ne s'agit pas uniquement d'une survivance de l'asile. Le travail en UMD diffère de l'unité ordinaire ; au point que certains le préfèrent. 
Cela fait des années que Nadine travaille au secrétariat de l'UMD. Avec Pascale, elles sont deux. Efficaces, leur professionnalisme contient ce qu'il faut de méfiance et de bonne volonté. Le bureau qu'elles occupent, au centre du sas d'entrée, les situe au cœur de leur fonction. Ce sont elles qui actionnent la lourde porte pour tous ceux qui viennent de l'extérieur sans badge. L'extérieur, c'est à dire les allées de l'hôpital. Ce sont elles également qui donnent accès à l'enceinte intérieure du bâtiment, derrières d'autres lourdes portes aveugles. De chez elles, on ne voit ni le jardin, ni les cours, ni les pavillons, ni les patients. On est loin de la vie des unités. On est dans un monde administratif où elles règnent, au milieu des bureaux des médecins, psychologues, cadres et autres soignants, et bien sûr de la petite cuisine.

Le travail administratif est dense et elles le font bien. Aux paperasses habituelles de la bureaucratie hospitalière s'ajoutent les obligations légales. Les allers et retours avec le préfet et avec les juges, les arrêtés d'admission et de sortie, les autorisations de visite de la famille, les négociations avec l'administration pénitentiaire, les transports sécurisés à organiser, les relations avec les secteurs d'origine à entretenir... Encore plus qu'ailleurs, tout ce qui se fait et se décide est encadré, autorisé, contrôlé, et tout est consigné.

De son poste d'observation, Nadine n'est finalement pas si loin de la vie de l'intérieur. Nadine est une charnière du système et elle est immergée dans sa violence. Elle a vu dans les dossiers les drames passés qui justifient l'admission des patients; elle a reçu les appels des secteurs en difficulté. Elle sait pourquoi les patients restent. Elle tape les lettres qui les concernent, tient le calendrier des attestations et certificats à jour. Les soignants lui racontent les anecdotes et événements qui surviennent dans les unités, quand ils traversent le sas. Elle écoute leurs plaintes. Elle entend les alertes.

Depuis son sas, elle est au cœur de la tension de l'unité et de la dureté des rapports, d'un côté comme de l'autre. Elle n'a pas l'air d'en tirer plaisir ou fascination, pas plus qu'elle ne donne l'impression d'en avoir peur. Elle est avenante, plutôt bienveillante. Elle est attachée aux patients, presque maternelle parfois. Elle est consciencieuse et garde son sens pratique. Est-ce qu'elle n'a pas envie, parfois, de revenir à un environnement moins violent, sans ces tensions de la vie quotidienne et ces pressions administratives? De rompre avec cette étanchéité entre le monde administratif et celui du soin. On croirait volontiers que cela use, d'être secrétaire dans cette unité où l'on est confrontée en permanence à ces situations extrêmes.

Pas du tout. Surtout pas. Nadine est catégorique. Elle garde un très mauvais souvenir de son expérience dans une unité de secteur, avec tous ces patients chroniques. Ce qui est bien, ici, m'explique-t-elle, c'est que les patients partent, toujours, à un moment donné. On a l'impression d'être efficace. Ça tourne. On échappe à cette sensation déprimante de traîner des années des patients qui ne vont jamais mieux. Je retiens mon objection. Cette efficacité est structurelle : l'UMD décide des admissions et le service d'hospitalisation d'origine est tenu de reprendre son patient, donc le turn-over est assuré. Par contre, rien ne dit que les patients vont mieux. On sait simplement qu'ils sont suffisamment calmés pour retourner dans leur service d'origine. Est-ce vraiment satisfaisant ? Surtout qu'ici, « calmé » veut dire « rentré dans le rang ». D’ailleurs, ce qu'on appelle sortie, c'est en réalité le transfert dans un autre service d'hospitalisation. Il semble inimaginable aujourd'hui de laisser le patient retourner à la vie ordinaire à la sortie d'UMD, en levant 
la mesure d'hospitalisation. Il y a quelques décennies, c'était pourtant assez fréquent, d'après les registres que j'ai consultés. Je ne dis rien à Nadine, car je pense à cette fatigue, à ce poids de l'ennui et de l'inertie des unités d'hospitalisations, ce sentiment du temps qui ne passe pas, ou si lentement, redoublé par la perspective de l'éternelle répétition du retour des patients chroniques. D’une certaine façon, je la comprends.

\section{Moderniser l'enfermement ? Reconsidérer la dangerosité dans le soin psychiatrique}

Ce que dit Nadine vient en appui d'une autre facette de ces unités, qu'on voit alors poindre, celle de leur spécialisation. Déjà en 1910, pour justifier d'ouvrir la première de ces unités, Henri Colin en appelait à la nécessité d'un traitement spécifique pour ces patients qu'il qualifiait alors « d'aliénés criminels » - c'est à dire malades avant tout, à la différence des « criminels aliénés ». Un siècle après, la question ressurgit dans le débat public, avec l'audition publique que mène en 2011 la Haute Autorité de Santé : il faut détecter et traiter la dangerosité psychiatrique, et ce dans des lieux de soins spécialisés. Les UMD sont des candidats naturels; il leur faut cependant définir les savoirs et pratiques qui feraient leur spécificité.

Dans l'UMD où j'enquête, j'observe ce mouvement. Peu à peu, au fil des entretiens et des observations, se dessinent les justifications qui permettent aux professionnels d'inscrire ces unités dans une logique de soin spécialisé. D’abord, les psychiatres se réfèrent à une définition contextuelle de la notion de patient dangereux, notamment au moment de l'admission. La dangerosité n'est pas intrinsèque à un individu ou à sa pathologie, mais résulte d'une conjonction de facteurs conduisant à une situation de risque. Parmi ces facteurs, l'environnement, y compris les modalités de prise en charge, jouent un rôle majeur. Ainsi, le fonctionnement de l'unité, en combinant enfermement et règles de vie très strictes, leur permet de caractériser une réponse clinique adaptée, qui associe dimensions thérapeutiques et de sécurité. Les psychiatres revendiquent également une expertise pharmacologique, appuyée sur la temporalité moins contrainte et le taux d'encadrement élevé. Ainsi, le soin spécialisé que revendiquent les psychiatres d'UMD reste solidement arrimé dans la psychiatrie générale : il vient pallier aux difficultés des services d'hospitalisation classique plutôt que traiter des pathologies spécifiques.

\section{La préoccupation sécuritaire}

En 2010, alors que j'assiste au processus de modernisation de l'héritage asilaire au sein des UMD existantes, le gouvernement annonce la création de quatre nouvelles unités. L'augmentation est historique. Quelques mois après, c'est la création d'un nouveau type d'unité qui est annoncée: les unités hospitalières spécialement aménagées (UHSA) destinées à hospitaliser les détenus. Neuf unités sont programmées, dont les cinq premières sont déjà financées. L'engagement politique est clair. Ces unités sont de nouvelles constructions - souvent situées dans l'enceinte des hôpitaux psychiatriques dont le nombre de lit a fondu ces dernières décennies. Leur sécurisation génère un coût important, en moyens matériels mais aussi en personnel - qui est plus nombreux et mieux payé que dans les unités ordinaires. Manifestement, en psychiatrie à cette période, c'est dans la sécurisation que va l'argent, et cette sécurisation prend la forme d'une spécialisation. Les détenus d'un côté, les patients difficiles de l'autre, les patients ordinaires enfin encore ailleurs. 


\section{Soin et sécurisation : vers les limites du thérapeutique}

La création des UHSA - alliée à l'augmentation des places en UMD - redéfinit les missions, la régulation et le fonctionnement de l'ensemble des unités psychiatriques sécurisées en France. Les détenus n'ont-ils plus leur place parmi les malades difficiles en UMD, puisque les UHSA leur sont destinées ? De quelles unités relèvent les personnes hospitalisées après avoir été déclarées pénalement irresponsables par la justice ? Les soignants d'UHSA sont-ils déchargés de la mission de sécurisation, dans la mesure où des surveillants de l'administration pénitentiaire sont présents? Les rapports entre psychiatrie, prison et justice se réorganisent, et avec eux les justifications de l'enfermement.

Ces unités sécurisées se distinguent de la psychiatrie ordinaire par leur recours à l'enfermement. Les psychiatres qui les développent y voient une occasion pour définir un soin spécialisé. Celui-ci diffère selon les unités. Dans les UHSA, la proximité avec l'administration pénitentiaire rend nécessaire de se distinguer de l'enfermement carcéral. Après des hésitations, les psychiatres responsables des unités choisissent plutôt de recentrer leur mission sur l'accès aux soins et la préservation de la dignité des détenus. Au contraire, les psychiatres travaillant en UMD revendiquent leur expertise en matière de sécurisation et de protection, marquant ainsi leur spécificité vis à vis des unités de soin ordinaires, mais sans pour autant donner de valeur thérapeutique à ces mesures.

\section{Hospitaliser n'est pas enfermer. La prise de conscience légale}

Des acteurs se saisissent de ces transformations du paysage des unités sécurisées et portent la question de l'enfermement en psychiatrie au-delà du cercle d'initiés. Comment concilier la vision de la psychiatrie de secteur, ouverte sur la communauté, avec le financement massif d'unités caractérisées par leur fermeture et la présence systématique de chambres d'isolement dans les services? La psychiatrie est-elle restée sécuritaire? Ces pratiques sont-elles compatibles avec la conception actuelle du soin psychiatrique? Respectent-elles les droits des personnes?

Des psychiatres et des associations, de familles et d'usagers de la psychiatrie font entendre leur voix. Certains, comme le Collectif des 39, défendent une psychiatrie humaniste, qui refuse l'enfermement. D'autres empruntent la voie du droit. Des militants et anciens usagers de la psychiatrie - au sein du Groupe Information Asiles (GIA) puis du Cercle de Réflexion et de Proposition d'Actions sur la psychiatrie (CRPA) mènent une véritable bataille juridique, enchaînant les recours contre les décisions médicales. Cette stratégie de long terme, qui s'appuie notamment sur les décisions des juridictions européennes, conduit, en 2011, à la réforme de la loi concernant les hospitalisations sans consentement. Chaque mesure sera contrôlée par un juge des libertés et de la détention. Certains mettent en cause la nature de ce contrôle et son efficacité. Peu importe, cette réforme renforce l'idée que l'hospitalisation sans consentement est bien une atteinte à la liberté d'aller et venir, un droit fondamental.

Les droits fondamentaux, de fait, se matérialisent en psychiatrie. En 2007, je rejoins un groupe de chercheurs réuni par le tout nouveau Contrôleur général des lieux de privation de liberté (CGLPL) et vois la démarche se construire. Le CGLPL, institué suite à 
une directive européenne, a pour mission de veiller au respect des droits fondamentaux dans ces lieux. Dès son entrée en fonction, Jean-Marie Delarue, décide d'inclure les hôpitaux psychiatriques à son périmètre qui compte déjà les prisons, les lieux de garde à vue et les centres de rétention. Pour lui, il faut prendre acte du fait que des personnes y sont, de fait, privées de leur liberté d'aller et venir.

\section{L'enfermement comme privation de liberté. Au nom de quoi?}

En adoptant ce parti-pris, le contrôleur général place les établissements psychiatriques sous le même impératif de contrôle que les établissements carcéraux, au regard des atteintes aux libertés qu'ils sont autorisés à effectuer. La procédure de contrôle repose sur des visites de plusieurs jours de l'équipe du CGLPL, qui rencontre un grand nombre d'acteurs et publie un compte-rendu détaillé de ses constats. Les rapports de visite sont rendus publics et dénoncent avec constance les atteintes aux droits fondamentaux constatées, sans masquer les réalités institutionnelles qui les favorisent parfois. Dans les rapports et avis qu'il produit régulièrement, le CGLPL porte le souci du respect des droits des personnes, y compris lorsqu'existent des raisons médicales aux décisions prises. Il illustre les façons très concrètes qui incarnent ce respect, ou son absence. Ainsi, indirectement, le CGLPL a fait peser sur les acteurs de la psychiatrie une pression au changement qui se fait sentir sur le moyen terme. En 2016 par exemple, lorsque Denys Robilliard propose de réglementer le recours à l'isolement et à la contention, la question fait débat. Cependant, plus personne n'affirme comme une évidence que l'enfermement fait partie intégrante du soin. A l'inverse, il arrive que l'argument du respect de certains droits fondamentaux soit avancé contre la suprématie de la décision médicale. Avec la mise en avant du consentement et la diffusion des droits fondamentaux, l'enfermement en psychiatrie apparaît avant tout comme une privation de liberté, autrement dit une atteinte à la liberté d'aller et venir.

L'enfermement a longtemps été considéré comme constitutif de la psychiatrie, dans un modèle thérapeutique associé à un lieu de soin, l'asile, et à un encadrement juridique spécifique. Pour en justifier, on intriquait le souci de sécurisation de l'extérieur, celui de protéger la personne et l'ambition thérapeutique. L'asile était aussi un refuge. Avec la réforme de la psychiatrie publique et l'évolution des pratiques de soin, l'enfermement est resté largement impensé, entre soin et sécurité. Puis, vers le milieu des années 2000, la place de l'enfermement a été redessinée, sous l'effet conjoint du développement d'unités sécurisées et du rôle croissant des droits fondamentaux dans la régulation de la relation de soin. La dimension positive de l'enfermement est largement délégitimée ; seule reste la justification sécuritaire. Paradoxalement, cette clarification aboutit parfois à dessiner des visions binaires, où l'enfermement s'oppose à la liberté, comme la contrainte au consentement, qui reflètent mal les dilemmes éthiques et pratiques que soulève le soin en psychiatrie. 
Les références à la littérature en sciences sociales et aux recherches mentionnées ne figurent pas dans le texte pour une meilleure lisibilité. Pour y accéder, le lecteur peut consulter :

Velpry L., Vidal-Naquet P. A., Eyraud B. (eds), 2018 Contrainte et consentement en santé mentale. Forcer, influencer, coopérer. Rennes, PUR, collection « Le sens social»

Velpry L, 2016 «Moderniser» l'enfermement en psychiatrie ? Le cas des unités pour malades difficiles, Sociétés contemporaines, 3 (n²103), pp. 65-90

Velpry L., Collectif Contrast, 2015, La création des UHSA : une nouvelle régulation de l'enfermement? Déviance et Société, vol.39, n4, pp. 429-453;

Velpry L., 2013, Violence et folie : dits et non-dits, Actualité et dossier en santé publique ADSP, $n^{\circ} 84$

Velpry L., Rhenter P., 2011, La réforme des hospitalisations psychiatriques sans consentement, Regards sur l'actualité, La Documentation Française, 10 (374), pp. 68-77

Velpry L., 2011, Maltraitance, dangerosité et souffrance au travail : formes et enjeux de la violence dans le contexte des soins en psychiatrie. In Rostaing C. et Laforgue D. (Eds.), Violences et institutions. Réguler, innover ou résister ?, Paris, CNRS Éditions, p. 29-44 